\title{
Guideline implementation in mental health: Current status and future goals
}

\author{
C. Barbui*, Guest Editor and M. Tansella, Editor
}

First published online 27 March 2012

According to the principles of evidence-based medicine, research findings should guide doctors when taking decisions in clinical practice. However, access and use of research findings may not be straightforward for most doctors in most countries of the world. In recent years, in order to overcome this global issue, new methodologies for aggregating, synthesizing and grading the quality of scientific evidence have progressively been developed, and approaches for creating clinical practice guidelines based on explicit assessments of the evidence base are nowadays commonly used in several fields of medicine, including mental healthcare (Barbui et al. 2010; Barbui \& Cipriani, 2011).

Surprisingly, however, while the pathway from evidence generation to evidence synthesis and guideline development is highly developed and quite sophisticated, the pathway from evidence-based guidelines to an evidence-based practice is much less developed (Barbui \& Tansella, 2011). In this issue of Epidemiology and Psychiatric Sciences three Editorials addressed these compelling questions from three different perspectives.

Anna Gagliardi reviewed the main concepts of guideline implementation and use to ascertain whether it is possible to derive suggestions for guiding research and practice in mental health (Gagliardi, 2012). A first interesting aspect is the concept of implementability, which refers to those characteristics that may enhance the uptake of guidelines by users. The content and format of guidelines, for example, may facilitate or impede their use, and current research has been focused to define the most relevant implementability elements, organized around an implementability framework. So far, the following elements have been identified: adaptability, usability, relevance, validity, applicability, communicability, resource implication, implementation and evaluation. The key idea, as suggested by Anna Gagliardi, is that addressing and taking into consideration these elements early in the initial phases of

* Address for correspondence: Professor C. Barbui, Department of Public Health and Community Medicine, Section of Psychiatry, Ospedale Policlinico 'G.B. Rossi', Piazzale L.A. Scuro 10, 37134 Verona, Italy.

(Email: corrado.barbui@univr.it) guideline development may significantly increase their implementability and uptake in practice. It would be relevant to apply this framework to the development and use of mental health guidelines, and formally test if this may indeed lead to better and sustained guideline implementation in mental healthcare.

A second aspect highlighted by Anna Gagliardi is that intense care should be devoted to the identification and selection of some interventions that may promote guideline use, including educational activities, social engagement, clinical support systems, incentives, audit and feedback exercises. The key aspect is that guideline implementation is meant to be iterative, in the sense that following implementation, guideline use and outcomes should be monitored, and the findings used to inform ongoing quality improvement efforts, as the ultimate goal of any implementation activity is continuous quality improvement.

Clearly, the difficult task is translating these concepts into operational protocols. Eivind Aakhus and colleagues, by presenting an implementation plan dealing with the management of depression in elderly patients, make a concrete effort in this direction (Aakhus et al. 2012). The key-word of their plan is 'tailored implementation', which refers to a flexible process where different implementation strategies are used depending on the varying nature of different recommendations. For example, organizational capacity to implement changes are critical for implementing a recommendation for collaborative care, but may be unimportant for implementing a recommendation against the first-line use of antidepressants in mild depression. Of note, to formally evaluate the feasibility of such an implementation strategy, a research design with random allocation has been developed, providing this way a paradigmatic example of how quality improvement in clinical practice may virtuously coexist with efforts to increase knowledge.

It should be recognized that the problem of translating knowledge into practice is deeply influenced by local variables, so for example the operational protocol described by Aakhus and colleagues may be feasible in Norway but might not work in a very diverse setting, such as a low-income mental health system. In his contribution Graham Thornicroft, who reviewed some 
peculiarities associated with guideline implementation in low- and middle-income countries (LAMIC) (Thornicroft, 2012), mentioned that WHO has recently produced an evidence-based intervention package with recommendations to facilitate care by the nonspecialist health care providers in low- and middle-income countries (Dua et al. 2011). Hopefully, this initiative will represent a step change opportunity to practitioners in LAMICs; however, no evidence exists on how this package should be implemented. Researchers and funders have tremendous responsibility in this context. We argue that future activities should be organized considering that the development of a new generation of implementation studies filling the gap between the production of knowledge and its uptake in practice is a pressing priority worldwide.

\section{Implementare Linee-Guida in Salute Mentale: Situazione Attuale e Obiettivi Futuri}

Secondo i principi della medicina basata sulle prove di efficacia le conoscenze scientifiche sono un aspetto rilevante del processo decisionale terapeutico in ambito sanitario. Le conoscenze scientifiche, tuttavia, non sono facilmente accessibili e fruibili, e per questo negli ultimi anni si sono sviluppate metodologie innovative per costruire raccomandazioni cliniche, basate sulle migliori conoscenze disponibili, che consentano di superare la barriera costituita dalla scarsa accessibilità delle evidenze scientifiche. Gli operatori sanitari potrebbero infatti utilizzare direttamente le raccomandazioni costruite sulla base delle evidenze senza la necessità di accedere alle evidenze stesse. Queste metodologie possono essere utilizzata per sviluppare raccomandazioni per gli operatori di un dipartimento di salute mentale, ma potrebbero essere anche impiegate per sviluppare raccomandazioni per un'ampia gamma di interventi o di patologie, da adottare ad un livello locale, regionale o anche nazionale (Barbui et al. 2010; Barbui \& Cipriani, 2011).

Sorprendentemente, mentre il percorso che collega la produzione di conoscenze alla sintesi di queste ultime sotto forma di raccomandazioni è stato oggetto di grande attenzione nella letteratura internazionale, il percorso che dovrebbe occuparsi di come le raccomandazioni vengono messe in pratica è molto meno sviluppato (Barbui \& Tansella, 2011). In questo numero di Epidemiology and Psychiatric Sciences tre Editoriali affrontano il tema proponendo tre prospettive diverse.

Il contributo di Anna Gagliardi fornisce i concetti principali sull'argomento, chiamato nella letteratura anglosassone guideline implementation (Gagliardi, 2012). Il punto chiave è quello di comprendere il concetto di implementability, ossia di quali siano le caratteristiche e i fattori che possano aumentare l'utilizzo delle raccomandazioni nella pratica clinica quotidiana. Il contenuto e il formato delle linee-guida, per esempio, sono fattori rilevanti, ma non sono gli unici. Attualmente, si ritiene che elementi determinanti per il trasferimento alla pratica dei concetti contenuti nelle linee-guida siano l'adattabilità, il grado di fruibilità, la rilevanza, la validità, l'applicabilità, la comunicabilità, le implicazioni in termini di uso di risorse, la modalità pratica di implementazione a livello locale, e gli aspetti valutativi dell'impatto delle raccomandazioni stesse. Si ritiene, come espresso nel contributo di Anna Gagliardi, che questi elementi dovrebbero essere considerati precocemente nelle fasi iniziali di costruzione delle raccomandazioni per ottimizzarne l'impatto in termini di implementability.

Un secondo aspetto evidenziato da Anna Gagliardi si riferisce alla identificazione e realizzazione di interventi orientati a promuovere l'utilizzo delle lineeguida. Tra questi, secondo i dati della letteratura, vi sono le iniziative formative e sociali che ruotano attorno ai contenuti delle linee-guida, lo sviluppo di sistemi di supporto clinico, gli incentivi, i sistemi di audit e feedback. La logica sottostante è quella secondo cui il processo di trasferimento dei contenuti delle linee-guida alla operatività pratica è di tipo iterativo, nel senso che ai programmi di implementazione dovrebbero seguire i programmi di monitoraggio, e i risultati del monitoraggio dovrebbero essere restituiti agli operatori in un'ottica di riflessione sulle proprie pratiche, e di ulteriore cambiamento. Tutto questo ha come fine ultimo il miglioramento della qualità della cura, secondo una logica di processo continuo e non puntiforme.

Certamente, l'aspetto più complicato è tradurre questi concetti in protocolli operativi. Il secondo contributo, preparato da Eivind Aakhus e collaboratori, è un esempio pratico di quanto enunciato teoricamente in precedenza (Aakhus et al. 2012). Gli autori presentano un programma per ottimizzare la gestione della depressione nell'anziano, e utilizzano come principio ispiratore il concetto della individualizzazione della implementazione. In pratica, si tratta di utilizzare strategie di implementazione diverse a seconda del tipo di raccomandazione. Per esempio, mentre per rendere operativa una raccomandazione sulla collaborazione tra medicina generale e setting specialistico è necessario un lavoro di ottimizzazione 
delle capacità organizzative di tutto il sistema sanitario, lo stesso lavoro non si applica, perché non rilevante, alla implementazione di una raccomandazione puntuale, per esempio una raccomandazione negativa sull'utilizzo di antidepressivi nei soggetti con depressione lieve. Vale la pena notare, in aggiunta, che per valutare l'impatto del programma di ottimizzazione della gestione della depressione nell'anziano gli autori hanno previsto uno studio formale che prevede la randomizzazione. Questo aspetto è degno di nota, a rimarcare che gli sforzi per il miglioramento continuo di qualità possono coesistere in modo virtuoso con gli sforzi tesi alla produzione di nuove conoscenze.

Naturalmente, va riconosciuto che la grande questione del trasferimento delle conoscenze dal mondo della ricerca a quello della pratica è fortemente influenzato da variabili di contesto. Il protocollo descritto da Aakhus e colleghi, per esempio, è certamente applicabile alla situazione norvegese dove è stato pensato, ma potrebbe non essere adatto ad un altro contesto di cura, per esempio quello di un paese a basso reddito. Il contributo di Graham Thornicroft pone l'accento proprio su questo tema, e analizza le peculiarità che i programmi di implementazione dovrebbero avere quando sono rivolti a sistemi sanitari economicamente sprovvisti di elevate risorse (Thornicroft, 2012). Graham Thornicroft menziona, a questo proposito, le raccomandazioni prodotte recentemente dalla OMS in tema di trattamento delle malattie psichiatriche (Dua et al. 2011), con l'auspicio che l'impatto possa essere massimo. Purtroppo, ad oggi, sono ancora scarsissimi i dati solidi sui quali poggiare i programmi di implementazione. In quest'ottica, il mondo della ricerca e di coloro che supportano economicamente i progetti di implementazione hanno responsabilità rilevanti. Abbiamo bisogno di organizzare e pianificare il futuro tenendo in considerazione il bisogno - urgente e globale - di una nuova generazione di studi che si occupino di individuare e caratterizzare gli elementi determinanti per il trasferimento alla pratica dei contenuti delle linee-guida.

\section{Declaration of interest}

None.

\section{References}

Aakhus E, Flottorp S, Oxman AD (2012). Implementing evidence based guidelines for managing depression in elderly patients: A Norwegian perspective. Epidemiology and Psychiatric Sciences.

Barbui C, Cipriani A (2011). What are evidence-based treatment recommendations? Epidemiology and Psychiatric Sciences 20, 29-31.

Barbui C, Dua T, van Ommeren M, Yasamy M, Fleischmann A, Clark N, Thornicroft G, Hill S, Saxena S (2010). Challenges in developing evidence-based recommendations using the GRADE approach: The case of mental, neurological, and substance use disorders. Plos Medicine 7, e1000322.

Barbui C, Tansella M (2011). Cochrane reviews impact on mental health policy and practice. Epidemiology and Psychiatric Sciences 20, 211-214.

Dua T, Barbui C, Clark N, Fleischmann A, Poznyak V, van Ommeren M, Yasamy MT, Ayuso-Mateos JL, Birbeck GL, Drummond C, Freeman M, Giannakopoulos P, Levav I, Obot IS, Omigbodun O, Patel V, Phillips M, Prince M, Rahimi-Movaghar A, Rahman A, Sander JW, Saunders JB, Servili C, Rangaswamy T, Unutzer J, Ventevogel P, Vijayakumar L, Thornicroft G, Saxena S (2011).

Evidence-based guidelines for mental, neurological, and substance use disorders in low- and middle-income countries: summary of WHO recommendations. PLoS Medicine 8, e1001122.

Gagliardi A (2012). Translating knowledge to practice: optimizing the use of guidelines. Epidemiology and Psychiatric Sciences in press.

Thornicroft G (2012). Evidence-based mental health care and implementation science in low- and middle-income countries. Epidemiology and Psychiatric Sciences in press. 